\title{
Time constrained liner shipping network design
}

\author{
Karsten, Christian Vad; Brouer, Berit Dangaard; Desaulniers, Guy; Pisinger, David
}

Published in:

Transportation Research. Part E: Logistics and Transportation Review

Link to article, DOI:

10.1016/j.tre.2016.03.010

Publication date:

2017

Document Version

Peer reviewed version

Link back to DTU Orbit

Citation (APA):

Karsten, C. V., Brouer, B. D., Desaulniers, G., \& Pisinger, D. (2017). Time constrained liner shipping network design. Transportation Research. Part E: Logistics and Transportation Review, 105, 152-162.

https://doi.org/10.1016/j.tre.2016.03.010

\section{General rights}

Copyright and moral rights for the publications made accessible in the public portal are retained by the authors and/or other copyright owners and it is a condition of accessing publications that users recognise and abide by the legal requirements associated with these rights.

- Users may download and print one copy of any publication from the public portal for the purpose of private study or research.

- You may not further distribute the material or use it for any profit-making activity or commercial gain

- You may freely distribute the URL identifying the publication in the public portal

If you believe that this document breaches copyright please contact us providing details, and we will remove access to the work immediately and investigate your claim 


\title{
Chapter 4
}

\section{Time Constrained Liner Shipping Network Design \\ with B.D. Brouer, G. Desaulniers, and D. Pisinger ${ }^{1}$}

\begin{abstract}
We present a mathematical model and a solution method for the liner shipping network design problem. The model takes into account coordination between vessels and transit time restrictions on the cargo flow. The solution method is an improvement heuristic, where an integer program is solved iteratively to perform moves in a large neighborhood search. To assess the effects of insertions/removals of port calls, flow and revenue changes are estimated for relevant commodities along with an estimation of the change in the vessel cost. Our improvement heuristic is applicable as a real-time decision support tool for a liner shipping company. It can be used to find improvements to the network when evaluating changes in operating conditions or testing different scenarios. Computational results on the benchmark suite Liner-lib are reported. For these instances we obtain insight about the optimal operational speed of the vessels, as well as statistics on which cargo reaches the destination within the requested time limit. Furthermore, we demonstrate that the algorithm can construct high-quality networks for most instances. Finally, we show how the algorithm can be extended to introduce a limit on the number of transshipments for each container.
\end{abstract}

\footnotetext{
${ }^{1}$ Karsten, C. V., Brouer, B. D., Desaulniers, G., and Pisinger, D. (2015). Time Constrained Liner Shipping Network Design. Submitted to: Transportation Research Part E special issue on "Computational Logistics at Work: Coordination and Control in Transport Logistics." and Brouer, B. D., Desaulniers, G., Karsten, C. V., and Pisinger, D. (2015). A matheuristic for the liner shipping network design problem with transit time restrictions. Computaional Logistics, volume 9335 of Lecture Notes in Computer Science, pages 195-208. Springer International Publishing.
} 


\subsection{Introduction}

The time constrained liner shipping network design problem, TCLSNDP, is a core planning problem faced by container carriers. The problem is to design a set of cyclic routes, services, for container vessels to provide transport for goods while respecting cargo travel time restrictions. The objective of the problem is to maximize the profit of the liner shipping company through the revenues gained from container transport taking into account the fixed cost of deploying vessels and the variable cost related to the operation of the routes and the handling cost of cargo transport. As a consequence of maximizing profits the liner shipping network design problem generally allows rejection of some commodities if deemed unprofitable.

Liner shipping companies offer a range of services that are operated according to a published schedule with a fixed frequency to make it easier for customers to plan ahead. Scheduling decisions refer to the temporal aspect of the vessel routings and include the timing of events along the entire round trip. A fleet of vessels is deployed to the services such that the capacity and speed is in accordance with the demand maintaining the desired frequency. Global carriers generally deploy vessels with similar characteristics to a service to reduce the complexity of the network design and corresponding schedules (Notteboom and Vernimmen, 2009; Stopford, 2009). The services give rise to a network of related ports. Containers, or more generally commodities, are transported through the network from port A to port B, and a transport may include the use of several services to connect between the origin and destination ports. The transits between services are referred to as transshipments and the transit time is the time used to transport a container from origin to destination. Each transport must respect a maximal transit time restriction of each individual commodity. In practice transit times vary from one day to several months and most containers are transshipped no more than twice corresponding to a feeder main line - feeder connection. However, some containers can be subject to up to five or six transshipments increasing the risk of delays and total handling time. Generally, customers prefer transports with no or few transshipments. The number of transshipments can vary based on the origin and destination regions. A network with direct connections between all serviced ports would offer low transit times and no transshipments, but at the same time it would be very expensive to operate for the carrier as most pairs of ports do not have enough container demand to fill a vessel. This illustrates the trade-off faced by liner shipping companies between the cost of networks versus transit time and/or number of transshipments offered to the customers. Providing low freight rates by minimizing the cost of the network is likely to result in prolonged transit times as exemplified in Karsten et al. (2015). Likewise designing a network to 
minimize transit times is likely to result in a very costly network since speed increases.

The main costs associated with the fleet include fuel cost, port and canal charges, and financing of vessels (this includes capital costs of acquiring or financing a vessel and the operational cost (OPEX) which includes crew, maintenance, and insurance). Stopford (2009) estimated the fuel cost to be $35-50 \%$ of a vessel's operational cost, capital cost to be 30-45 \%, OPEX to be 6-17\%, and port cost to be 9-14\%. This obviously depends on the fuel price and general economic environment. The cargo handling cost is calculated from the load and unload cost at the origin and destination ports and the cost associated with transshipments at intermediate ports. In addition to this, there are costs for the customer associated with owning or leasing containers. The load and unload costs do not depend on the routing of the container, whereas the transshipment cost does. Revenues are obtained by transporting cargo through the network and varies based on the type of cargo and the level of service offered.

Recent literature on the liner shipping network design problem, LSNDP, allows arbitrary transit times for all commodities (Brouer et al. $2014 b$, Liu et al. 2014; Wang and Meng, 2014; Mulder and Dekker, 2014, Brouer et al., 2014a Plum et al., 2014; Reinhardt and Pisinger, 2012, Gelareh et al., 2010, Agarwal and Ergun, 2008) although it is generally acknowledged that transit times are decisive for the competitiveness of the network design, e.g. Brouer et al. (2014a). Initial work to construct a multi-criteria objective function is presented in Álvarez (2012) that considers a bi-linear expression for the inventory cost of the cargo on board vessels, but the level of service calculations are not computationally tractable in the already very complex liner shipping network design models. However, the inventory cost of commodities on board vessels is only indirectly a concern to the carrier, when excessive transit times result in the customers switching to a different carrier. Hence, the carriers concern is to ensure a maximal transit time corresponding to the market level of service. Wang and Meng (2014) introduce deadlines on commodities in a non-linear, non-convex mixed-integer programming (MIP) formulation of liner shipping network design with transit time restrictions. As a consequence the model does not allow transshipment of cargo, which is another common trait of the liner shipping network design problem.

Brouer et al. (2014b) develop a matheuristic for the LSNDP. The matheuristic is an improvement heuristic according to the categorization in the survey on matheuristics by Archetti and Speranza (2014) meaning that an integer program is used as a move operator. The present paper extends the method of Brouer et al. (2014b) to include transit times. Álvarez (2012) presents math- 
ematical expressions for the inventory cost of containers during transport. No computational results are reported as the mathematical expressions are not easily incorporated into existing models of the LSNDP. In Wang et al. (2013) an integer program for deciding minimum cost container paths for a single OD pair respecting transit time and cabotage restrictions is considered. Karsten et al. (2015) present a column generation algorithm for a time constrained multicommodity flow (MCF) problem applied to a liner shipping network. A resource constrained shortest path problem is solved for each origin using a specialized label setting algorithm. Different topologies of graphs for liner shipping networks are presented. Computational results for solving the MCF problem with and without transit times on global-sized liner shipping networks are reported. The solution times for the time constrained MCF problem is comparable to solving the MCF problem without transit time restrictions. The algorithm of Karsten et al. (2015) is used in the matheuristic presented in this paper for evaluating a given network during the search. A liner shipping network design problem considering transit time restrictions is presented in Wang and Meng (2014). The model excludes transshipments between services. The problem is proven to be NP-hard and is formulated as a non-linear, non-convex mixed integer program. A column-generation-based heuristic is developed and a case study is presented for a network of 12 main ports on the Asia-Europe trade lane with three different vessel classes. The model is suggested as an aid to planners in a liner shipping company and the case study provides high-quality network suggestions and important insights to assist the planners. The authors suggest incorporation of transshipments along with transit time restrictions as an area of future research.

Meng et al. (2014) and Christiansen et al. (2013) provide broader reviews of recent research on routing and scheduling problems within liner shipping.

In this paper we present a capacitated multicommodity network design formulation for the TCLSNDP allowing for an arbitrary number of transshipments and enabling restrictions on transit time of individual commodities. We propose an adaptation of the matheuristic of Brouer et al. (2014b) to show that it is possible to incorporate the transit time restrictions in a heuristic context. Furthermore, we show that it is tractable to incorporate a limit on the number of transshipments for each commodity. The benchmark instances presented in Brouer et al. (2014a), Liner-lib, are used for the computational results of this paper. The benchmark instances contain maximum transit time for all OD pairs.

The rest of this paper is organized as follows: Section 4.2 introduces our mathematical model. Section 4.3 extends the IP used as a move operator in Brouer 
et al. (2014b) to also consider transit times. Section 4.4 reports computational results for the benchmark instances. Section 4.5 shows the sensitivity to the provided transit times and Section 4.6 extends the algorithm and results with a limit on the number of transshipments. We end the paper by drawing conclusions and discussing extensions in Section 4.7

\subsection{Mathematical Model for the TCLSNDP}

In the following we introduce the notation used to formulate the TCLSNDP mathematically. An instance of the TCLSNDP consists of the following sets:

- $P$ : Set of ports with an associated port call cost $c_{p}^{e}$, unit (un)load cost $c_{U}^{p}, c_{L}^{p}$, unit transshipment $\operatorname{cost} c_{T}^{p}$ and berthing time $b_{p}$ spent on a port call.

- $K$ : Set of demands, where each demand has an origin $O_{k} \in P$, a destination $D_{k} \in P$, a quantity, $q_{k}$, a revenue per unit, $z_{k}$, a reject penalty per unit $\tilde{z}_{k}$ and a maximal transit time, $t_{k}$.

- $E$ : Set of vessel classes with specifications for the weekly charter rate, $f_{e}$, capacity $U_{e}$, minimum $\left(v_{\min }^{e}\right)$ and maximum $\left(v_{\max }^{e}\right)$ speed limits in knots per hour, fuel consumption $g_{v}^{e}$ as a function of the speed, and fuel consumption $h^{e}$ per hour, when the vessel is idle at ports. There are $N_{e}$ vessels available of class $e \in E$. The price for one metric ton of fuel is denoted $c_{B}$.

- D: Matrix of the direct distances $d_{i j}^{e}$ between all pairs of ports $i, j \in P$ and for all vessel classes $e \in E$. The distance may depend on the vessel class draft as the Panama canal is draft restricted. Along with $d_{i j}^{e}$ follows an indication of the cost $l_{i j}^{e}$ associated with a possible traversal of a canal.

A solution to the TCLSNDP is a subset of the set of feasible services $S$. A service consists of a set of ports $P^{\prime} \subseteq P$, a number of vessels, and an average sailing speed. A service is cyclic but may be non-simple, that is, ports can be visited more than once. In this model we allow a single port to be visited twice, yielding a so-called butterfly route. A weekly frequency of port calls is obtained by deploying multiple vessels to a service. Let $e(s) \in E$ be the vessel class assigned to a service $s$ and $n_{e(s)}$ the number of vessels of class $e(s)$ required to maintain a weekly frequency. A round trip may last several weeks but due 
to the weekly frequency exactly one round trip is performed every week. Let $v_{s}$ be the service speed in nautical miles per hour.

The mathematical model of the TCLSNDP relies on a set of service variables and a path flow formulation of the underlying time constrained MCF problem. To describe the service network of the TCLSNDP, we define $F^{s}$ to be the port sequence $p_{1}^{s}, p_{2}^{s}, \ldots, p_{m}^{s}$ for the service $s \in S$. Let $|s|$ denote the number of unique ports in a service $s \in S$ and $\left|F^{s}\right|=m$ the number of port calls in $s$.

Furthermore we define a directed graph, $G(V, A)$, with vertices $V$ and arcs A. $V=V_{P} \cup V_{R}$ is the set of vertices, where $V_{P}$ is the subset of vertices representing the unique ports $p \in P$, and $V_{R}$ is the subset of service vertices representing all port calls by all services. $V_{R}=\bigcup_{s \in S} V_{F^{s}}$ and $V_{F^{s}}$ is the subset of vertices representing the port calls $p_{1}^{s}, p_{2}^{s}, \ldots, p_{m}^{s}$ of service $F^{s}, s \in S . p(v)$ is a function mapping a vertex $v \in V_{R}$ (i.e., a port call) to its actual port $p \in P$. The set of arcs in the graph can be divided into (un)load arcs, transshipment arcs, sailing arcs, and forfeited arcs, i.e. $A=A_{L} \cup A_{U} \cup A_{T} \cup A_{S} \cup A_{K}$. These sets are formally defined below and we associate with each arc $a \in A$ a cost $c_{a}$, traversal time $t_{a}$, and capacity $C_{a}$.

- $A_{L}=\left\{(p, v) \mid p \in V_{P}, v \in V_{F^{s}}\right\}$ and $A_{U}=\left\{(v, p) \mid v \in V_{F^{s}}, p \in V_{P}\right\}$ are respectively the sets of loading/unloading arcs representing a departure/arrival at port $p$ visited in $F^{s}, c_{a}=c_{L}^{p}$, and $c_{a}=c_{U}^{p}$ is the (un)loading cost for a container at the associated port $p \in V_{P}, t_{a}=0$, and $C_{a}$ is unlimited.

- $A_{T}=\left\{(v, u) \mid v \in V_{F^{s}}, u \in V_{F^{s^{\prime}}}\right\}$ is the set of transshipment arcs representing a transshipment between services $F^{s}$ and $F^{s^{\prime}}$ defined for every pair $(v, u)$ where $p(v)=p(u), c_{a}=c_{T}^{p}$ is the transshipment cost for a container at the associated port $p \in V_{P}, t_{a}$ is the transshipment time, and $C_{a}$ is unlimited.

- $A_{S}=\left\{(v, u) \mid s \in S, v, u \in V_{F^{s}}, v=p_{h}^{s}, u=p_{((h+1) \bmod m)}^{s}\right\}$ is the set of sailing arcs representing a sailing between two consecutive port calls $v$ and $u$ in $F^{s}, c_{a}=0$ as sailing costs are directly incurred by the vessels, $t_{a}=d_{u v} / v_{s}+b_{v}$ meaning the time in hours to traverse the edge plus the berthing time at the arriving port for each sailing, and $C_{a}=U_{e(s)}$.

- $A_{K}=\left\{(v, u) \mid v, u \in V_{P}, \exists k \in K: O_{k}=v \wedge D_{k}=u\right\}$ is the set of forfeiting arcs representing a rejection of transporting the cargo $k$ between $v$ and $u$ in $P, c_{a}=\tilde{z}_{k}+z_{k}$ is the penalty associated with rejecting the cargo $k$, $t_{a}=t_{k}$ is the maximum transit time for $k$, and $C_{a}=q_{k}$. 
We use the path flow formulation of the time constrained MCF problem as described in Karsten et al. (2015). Let $\Omega_{k}$ be the set of all feasible paths for commodity $k$ including forfeiting the cargo. Let $\Omega(a)$ be the set of all paths using arc $a \in A$. The cost of a path $\rho$ is denoted as $c_{\rho}$ and it includes the revenue obtained by transporting one unit of commodity $k$ sent along path $\rho \in \Omega_{k}$. The real nonnegative variable $x_{\rho}$ denotes the amount of commodity $k$ sent along the path. Let the weekly cost of a service be $c_{s}=n_{e(s)} f_{e(s)}+$ $\sum_{(i, j) \in A_{S}}\left(c_{B}\left(h^{e(s)} b_{p}+g_{v(s)}^{e(s)} d_{i j}^{e(s)}\right)+c_{j}^{e(s)}+l_{i j}^{e(s)}\right)$ accounting for fixed cost of deploying the vessel and the variable cost in terms of the fuel and port call cost of one round trip. Define binary service variables $y_{s}$ indicating the inclusion of service $s \in S$ in the solution.

Then the TCLSNDP can be formulated as the following mixed integer program:

$$
\begin{aligned}
& \min \quad \sum_{s \in S} c_{s} y_{s}+\sum_{k \in \mathbf{K}} \sum_{\rho \in \Omega_{k}} c_{\rho} x_{\rho} \\
& \text { s.t. } \quad \sum_{\rho \in \Omega_{k}} x_{\rho}=q_{k} \quad k \in K \\
& \sum_{\rho \in \Omega(a)} x_{\rho}-U_{e(s)} y_{s} \leq 0 \quad s \in S, a \in A_{S} \\
& \sum_{s \in S: e(s)=e} n_{e(s)} y_{s} \leq N_{e} \quad e \in E \\
& x_{\rho} \in \mathbb{R}^{+} \\
& \rho \in \Omega_{k}, k \in K \\
& y_{s} \in\{0,1\} \\
& s \in S
\end{aligned}
$$

The objective 4.1 minimizes cumulative service and cargo transportation cost. As the cargo transportation cost includes the revenue of transporting the cargo this is equivalent to maximizing profit. The cargo flow constraints (4.2) along with non-negativity constraints 4.5 ensure that all cargo is either transported or forfeited. The capacity constraints 4.3 link the cargo paths with the service capacity installed in the transportation network. The fleet availability constraints 4.4 ensure that the selected services can be operated by the available fleet. Finally, constraints (4.6) define the service variables as binary.

The mathematical model extends the problem description of the LSNDP presented in Brouer et al. (2014a) to handle transit times. The model enforces a weekly frequency resulting in a weekly planning horizon. The path flow formulation of the MCF problem considers transit time restrictions in the definition 
of a feasible path for a given commodity. Column generation is applied for solving the path flow formulation of the MCF problem, where reduced cost columns are generated by solving a shortest path problem. Introducing transit time restrictions changes the sub-problem to a resource constrained shortest path problem and thus the complexity of the sub-problem becomes NP-hard. The label setting algorithm from Karsten et al. (2015) is used to solve the cargo routing problem with transit time restrictions during the execution of our algorithm.

In the TCLSNDP the sailing speed is decisive for the cost of a given service as well as the feasible solution space of the multicommodity flow problem. The majority of all commodities are subject to transshipments and transit time may depend on the choice of speed on multiple services. As a consequence lowering the speed to reduce the cost of a service may make existing cargo routings infeasible due to an increase in transit times. Likewise, increasing speed may result in increased flow in the network as the set of feasible paths increase, but at the same time it will increase the cost of service through the additional fuel burn. The service variables of (4.1)-(4.6) are defined for an average speed on all sailings on a round trip and assume a fixed weekly frequency. Hence, the resulting speed and cost change from in- or de-creasing by one vessel may be quite significant. However, the proposed algorithm does not optimize speeds of the individual sailings. The feasible deployment of vessels to maintain weekly frequency will be limited by the minimum and maximum speed.

\subsection{Algorithm}

The algorithm presented in this paper is an extension of the matheuristic for the LSNDP presented in Brouer et al. (2014b). The algorithm proposed in Brouer et al. (2014b) uses a greedy knapsack based construction heuristic to create an initial set of services, S. Then the core of the matheuristic is executed iteratively to try to improve these using a MIP for each service. The algorithm terminates either when no profitable moves can be found or when a computational time limit is reached. We use the same overall framework in the following and a detailed description and flow chart of the algorithm can be found in Brouer et al. (2014b). The central component in the latter matheuristic is an improvement heuristic, where an integer program is solved as a move operator in a large neighborhood search. The integer program is iteratively solved for a single service using estimation functions for changes in the flow due to insertions and removals of port calls in the service investigated. The solution of the integer program provides a set of moves in the composition of port calls and 
fleet deployment. Flow changes and the resulting change in the revenue are estimated by solving a series of resource constrained shortest path problems on the residual graph of the current network. This is done for relevant commodities to the insertion/removal of a port call along with an estimation of the change in the vessel related cost with the current fleet deployment.

Given a total estimated change in revenue of $r e v_{i}$ and port call cost of $c_{i}^{e(s)}$ Figure $4.1 \mathrm{a}$ illustrates estimation functions for the change in revenue $\left(\Theta_{i}^{s}\right)$ and duration increase $\left(\Delta_{i}^{s}\right)$ for inserting port $i$ into service $s$ controlled by the binary variable $\gamma_{i}$. The duration controls the number of vessels needed to maintain a weekly frequency of service. Figure $4.1 \mathrm{~b}$ illustrates the estimation functions for the change in revenue $\left(\Upsilon_{i}^{s}\right)$ and decrease in duration $\left(\Gamma_{i}^{s}\right)$ for removing port $i$ from service $s$ controlled by the binary variable $\lambda_{i}$. Insertions/removals will affect the duration of the service in question and hence the needed fleet deployment modeled by the integer variable $\omega_{s}$ representing the change in the number of vessels deployed.

(a) Blue nodes are evaluated for insertion corresponding to variables $\gamma_{i}$ for the set of ports in the neighborhood $N^{s}$ of service $s$.

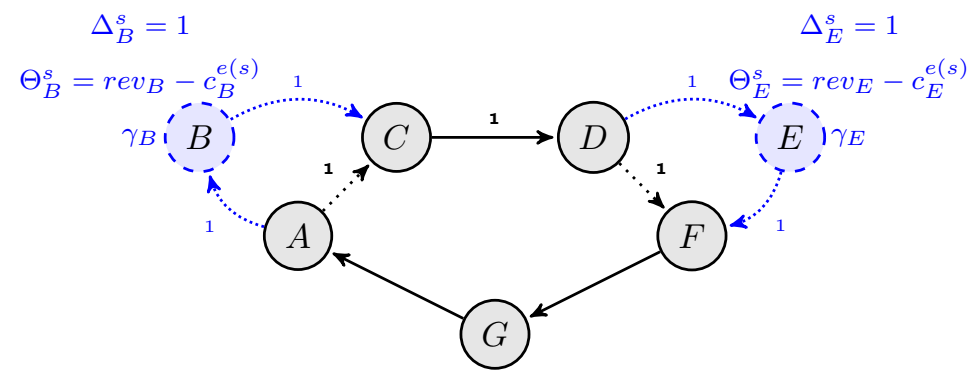

(b) Red nodes are evaluated for removal corresponding to variables $\lambda_{i}$ for the set of current port calls $F^{s}$ on service $s$.

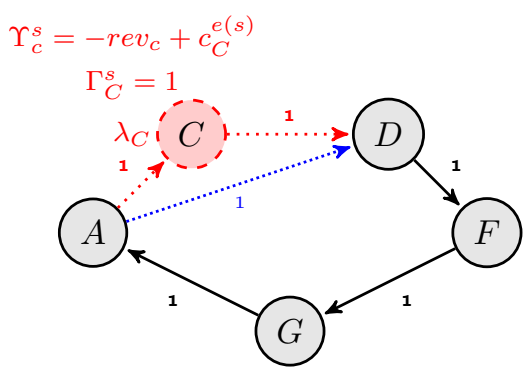

Figure 4.1: The estimation functions for insertion and removal of port calls. 


\subsubsection{Revenue Loss due to Transit Time Changes}

For considering the transit time in the IP, it is necessary to estimate how insertions and removals of port calls will affect the duration of the existing flow on the service. This means that existing flow must be estimated to have sufficient slack in transit time for the insertions performed or alternatively, existing flow will result in a loss of revenue if it cannot be rerouted within the available transit time on a different path. Figure 4.2 illustrates a case of a path with the associated variable in the current basis of the MCF model. When inserting port $B$ on its path it becomes infeasible due to transit time restrictions.

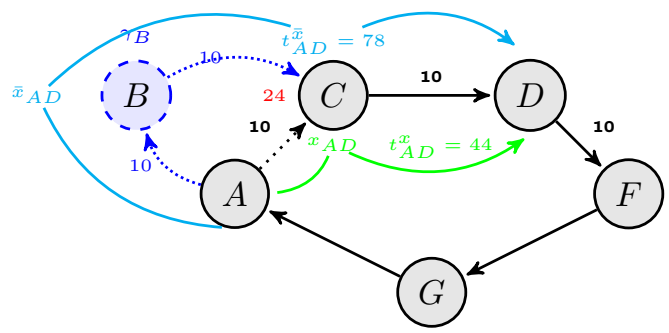

Figure 4.2: Insertions/removals affect transit time of the flow. Commodity $k_{A D}$ has a maximum transit time of 48 hours and the insertion of port call $\gamma_{B}$ will make the path infeasible.

In order to account for the transit time restrictions of the current flow, constraints 4.14 are added to the IP and a penalty, $\zeta_{x}$ corresponding to losing the cargo, is added to the objective if the transit time slack for an existing path becomes negative. This is handled through the variable $\alpha_{x}$, where $x$ refers to a path variable with positive flow in the current solution and $s_{x}$ refers to the current slack time according to the transit time restrictions of the associated path. For ease of reading, Table 4.1 gives an overview of additional sets, constants, and variables used in the IP. 


\begin{tabular}{ll} 
Sets & \\
\hline$N^{s}$ & Set of neighbors (potential port call insertions) of $s$. \\
$X^{s}$ & Set of path variables on service $s$ in current flow solution with pos. flow. \\
$N^{x} \subseteq N^{s}$ & Subset of neighbors inserted in current path of variable $x \in X^{s}$. \\
$F^{x} \subseteq F^{s}$ & Subset of port calls on current path of variable $x \in X^{s}$. \\
$L_{i}$ & Lock set for port call insertion $i \in N^{s}$ or port call removal $i \in F^{s}$. \\
\hline Constants & \\
\hline$Y_{s}$ & Distance of the route associated with $s$. \\
$K_{s}$ & Estimated average speed of the service $s$. \\
$M_{e}(s)$ & Number of undeployed vessels of class $e$ in the current solution. \\
$I_{s}$ & Maximum number of insertions allowed in $s$. \\
$R_{s}$ & Maximum number of removals allowed in $s$. \\
$\Delta_{i}^{s}$ & Estimated distance increase if port call $i \in N^{s}$ is inserted in $s$. \\
$\Gamma_{i}^{s}$ & Estimated distance decrease if port call $i \in F^{s}$ is removed from $s$. \\
$\Theta_{i}$ & Estimated profit increase of inserting port call $i \in N^{s}$ in $s$. \\
$\Upsilon_{i}$ & Estimated profit increase of removing port call $i \in F^{s}$ from $s$. \\
$\zeta_{x}$ & Estimated penalty for cargo lost due to transit time. \\
$s_{x}$ & Slack time of path variable $x$. \\
\hline Variables & \\
\hline$\lambda_{i}$ & 1 if port call $i \in F^{s}$ is removed from $s, 0$ otherwise. \\
$\gamma_{i}$ & 1 if port call $i \in N^{s}$ is inserted in $s, 0$ otherwise. \\
$\omega_{s} \in \mathbb{Z}$ & Number of vessels added (removed if negative) to $s$. \\
$\alpha_{x}$ & 1 if transit time of path variable $x \in X^{s}$ is violated, 0 otherwise. \\
&
\end{tabular}

Table 4.1: Overview of sets, constants, and variables used in the IP.

Given this notation, the IP is:

$$
\max \sum_{i \in N^{s}} \Theta_{i} \gamma_{i}+\sum_{i \in F^{s}} \Upsilon_{i} \lambda_{i}-f_{e(s)} \omega_{s}-\zeta_{x} \alpha_{x}
$$

Subject to:

$$
\begin{array}{ll}
\frac{Y_{s}}{K_{s}}+\sum_{i \in F^{s}} b_{p(i)}+\sum_{i \in N^{s}}\left(\frac{\Delta_{i}^{s}}{K_{s}}+b_{p(i)}\right) \gamma_{i}-\sum_{i \in F^{s}}\left(\frac{\Gamma_{i}^{s}}{K_{s}}+b_{p(i)}\right) \lambda_{i} \leq 168 \cdot\left(n_{e}(s)+\omega_{s}\right) \\
\omega_{s} \leq M_{e(s)} \\
\sum_{i \in N^{s}} \gamma_{i} \leq I_{s} & \\
\sum_{i \in F^{s}} \lambda_{i} \leq R_{s} & \\
\sum_{j \in L_{i}} \lambda_{j} \leq\left|L_{i}\right|\left(1-\gamma_{i}\right) & i \in N^{s} \\
\sum_{j \in L_{i}} \lambda_{j} \leq\left|L_{i}\right|\left(1-\lambda_{i}\right) & i \in F^{s}
\end{array}
$$




$$
\begin{aligned}
& \sum_{i \in N^{x}}\left(\frac{\Delta_{i}^{s}}{K_{s}}+b_{p(i)}\right) \gamma_{i}-\sum_{i \in F^{x}}\left(\frac{\Gamma_{i}^{s}}{K_{s}}+b_{p(i)}\right) \lambda_{i}-U B \alpha_{x} \leq s_{x} \quad x \in X^{s} \\
& \lambda_{i} \in\{0,1\}, i \in F^{s} \quad \gamma_{i} \in\{0,1\}, i \in N^{s} \quad \alpha_{x} \in\{0,1\}, x \in X^{s} \\
& \omega_{s} \in \mathbb{Z}, s \in S
\end{aligned}
$$

The objective function 4.7 maximizes the estimated profit increase obtained from removing and inserting port calls, accounting for the estimated change of revenue, transshipment cost, port call cost and fleet cost. As opposed to the IP proposed in Brouer et al. (2014b) the change in revenue may be related to not transporting cargo for which the path duration is estimated to exceed the transit time of the commodity. The number of vessels needed on the service (assuming a weekly frequency) after insertions/removals is estimated in constraint 4.8 accounting for the change in the service time given the current speed $K_{s}$. Constraint (4.9) ensures that the solution does not exceed the available fleet of vessels. Note that $\omega_{s}$ does not need to be bounded from below by $-n_{e(s)}$ because it is not allowed to remove all port calls. Constraints 4.10 and (4.11) limit the number of port call insertions and removals to minimize the error in the computed estimates. The set of port calls affected by an insertion or a removal is fixed by the lock set constraints $(4.12)$ and $(4.13)$, respectively. Finally, constraints (4.14) activate the estimated penalty for lost cargo due to an estimated violation of the transit time for the commodity on this particular path.

\subsection{Computational Results}

The matheuristic was tested on the benchmark suite Liner-lib described in Brouer et al. (2014a). The instances can be found at http://www.liner-lib.org. Table 4.2 gives an overview of the instances. We have revised the transit time restrictions for a small number of the origin-destination pairs in order to meet critical transit times as our model operates with average sailing speeds. The pairs where the transit times have been revised are those that cannot be satisfied by a direct sailing at 14 knots. The number of revised pairs is 6,15 , 106, and 32 for WAF, Pacific, WorldSmall, and AsiaEurope respectively. They have been revised according to the most recent published liner shipping transit times.

The matheuristic has been coded in $\mathrm{C}++$ and run on a linux system with an Intel $(R) X e o n(R) X 5550 \mathrm{CPU}$ at $2.67 \mathrm{GHz}$ and $24 \mathrm{~GB}$ RAM. The algorithm is set to terminate after the time limits imposed in Brouer et al. (2014a). 


\begin{tabular}{|c|c|c|c|c|c|c|c|}
\hline $\begin{array}{l}\text { Single- } \\
\text { hub }\end{array}$ & Instance and description & $|P|$ & $|K|$ & $E \mid$ & $\min v$ & $\max v$ & t.l. \\
\hline \multirow[t]{2}{*}{$\begin{array}{l}\text { Single- } \\
\text { hub }\end{array}$} & $\begin{array}{l}\text { Baltic Baltic sea, Bremerhaven } \\
\text { as hub }\end{array}$ & 12 & 22 & 2 & 5 & 7 & 300 \\
\hline & $\begin{array}{l}\text { WAF West Africa, Algeciras as } \\
\text { hub }\end{array}$ & 19 & 38 & 2 & 33 & 51 & 900 \\
\hline $\begin{array}{l}\text { Multi- } \\
\text { hub }\end{array}$ & $\begin{array}{l}\text { Mediterranean Mediter- } \\
\text { ranean, Algeciras, Tangier, and } \\
\text { Gioia Tauro as hubs }\end{array}$ & 39 & 369 & 3 & 15 & 25 & 1,200 \\
\hline Trade- & Pacific (Asia-US West) & 45 & 722 & 4 & 81 & 119 & 3,600 \\
\hline lane & $\begin{array}{l}\text { AsiaEurope Europe, Middle } \\
\text { East and Far east regions }\end{array}$ & 111 & $=000$ & 6 & 140 & 212 & 14,400 \\
\hline World & $\begin{array}{l}\text { Small } 47 \text { Main ports worldwide } \\
\text { identified by Maersk Line }\end{array}$ & 47 &, 764 & 6 & 209 & 317 & 10,800 \\
\hline
\end{tabular}

Table 4.2: The instances of the benchmark suite with indication of the number of ports $(|P|)$, the number of origin-destination pairs $(|K|)$, the number of vessel classes $(|E|)$, the minimum and maximum number of vessels (min $v$ and max $v)$, and the solution time limit in seconds (t.l.).

We fix the berthing time, $b_{p}$, to 24 hours for all ports as in Brouer et al. (2014a) and the transshipment time, $t_{a}$, is fixed to 48 hours for every connection. If a schedule was considered the inter-service transshipment time could be calculated based on arrival and departure times.

\subsubsection{Computational Results for Liner-lib}

Table 4.3 shows that the algorithm can find profitable solutions (negative objective values) for Baltic, WAF, WorldSmall and AsiaEurope. Pacific is unprofitable although both fleet deployment and transport percentage is high. In most instances except the Mediterranean around $85 \%$ to $95 \%$ of the available cargo is transported on average. At the same time as little as $80 \%$ of the fleet in terms of volume is utilized suggesting that further improvements may be achievable as the larger instances all terminate due to the imposed computational time limits. For the smaller instances the stopping criterion of the embedded simulated annealing procedure is fulfilled before the time limit is reached.

Table 4.4 shows that most services operate relatively close to their design speed for the smaller classes, apart from the WorldSmall instances where average ser- 


\begin{tabular}{lrrrrr}
\hline \multirow{2}{*}{ Instance } & Objective & \multicolumn{2}{c}{ Deployment } & Flow & CPU time \\
\cline { 2 - 6 } & $\mathbf{Z}(\mathbf{7})$ & $\mathbf{D}(\mathbf{v})$ & $\mathbf{D}(|E|)$ & $\mathbf{T}(\mathbf{v})$ & $(\mathbf{S})$ \\
\hline Best - Baltic & & $(\%)$ & $(\%)$ & $(\%)$ & \\
Average Baltic & $\mathbf{- 1 4 , 0 5 0}$ & $\mathbf{1 0 0 . 0}$ & $\mathbf{1 0 0 . 0}$ & $\mathbf{8 7 . 4}$ & $\mathbf{1 0 1}$ \\
\hline Best - WAF & 74,480 & 100.0 & 100.0 & 86.7 & 108 \\
Average WAF & $\mathbf{5 . 5 9} \cdot \mathbf{1 0}^{\mathbf{6}}$ & $\mathbf{8 3 . 3}$ & $\mathbf{8 5 . 7}$ & $\mathbf{9 7 . 0}$ & $\mathbf{2 5 5}$ \\
\hline Best - Med & $\mathbf{2 . 4 2} \cdot 10^{6}$ & 83.3 & 85.2 & 94.3 & 354 \\
Average Med & $2.70 \cdot 10^{6}$ & 90.5 & 94.0 & 78.9 & 737 \\
\hline Best - Pacific & $\mathbf{3 . 8 1} \cdot \mathbf{1 0}^{\mathbf{6}}$ & $\mathbf{9 5 . 5}$ & $\mathbf{9 6 . 0}$ & $\mathbf{9 4 . 7}$ & time \\
Average Pacific & $4.62 \cdot 10^{6}$ & 92.2 & 90.0 & 92.1 & time \\
\hline Best - WorldSmall & $\mathbf{- 3 . 1 8} \cdot \mathbf{1 0}^{\mathbf{7}}$ & $\mathbf{8 1 . 2}$ & $\mathbf{8 9 . 4}$ & $\mathbf{9 0 . 7}$ & time \\
Average WorldSmall & $-2.80 \cdot 10^{7}$ & 81.9 & 88.4 & 90.2 & time \\
\hline Best - AsiaEurope & $-\mathbf{1 . 7 6} \cdot \mathbf{1 0}^{\mathbf{7}}$ & $\mathbf{8 7 . 1}$ & $\mathbf{9 5 . 5}$ & $\mathbf{9 0 . 7}$ & time \\
Average AsiaEurope & $-1.45 \cdot 10^{7}$ & 84.8 & 92.3 & 88.4 & time \\
\hline
\end{tabular}

Table 4.3: Best and Average of 10 runs. Weekly profit (objective value) $\mathbf{Z}(7)$; percentage of fleet deployed: as a percentage of the total volume $\mathbf{D}(\mathbf{v})$, and as a percentage of the number of ships $\mathbf{D}(|E|) . \mathbf{T}(\mathbf{v})$ is the percentage of total cargo volume transported and $(\mathbf{S})$ is the execution time in CPU seconds; time means the solution time limit given in Table 4.2 has been reached.

\begin{tabular}{lrrrrrr}
\hline Instance & F450 & F800 & P1200 & P2400 & Post P & Super P \\
\hline Baltic & 10.8 & 13.7 & & & & \\
WAF & 11.5 & 13.2 & & & & \\
Med & 11.9 & 13.7 & 13.9 & & & \\
Pacific & 11.6 & 14.3 & 16.4 & 17.9 & & \\
WorldSmall & 12.9 & 15.2 & 17.3 & 19.4 & 19.4 & 17.1 \\
AsiaEurope & 11.6 & 13.9 & 16.8 & 17.9 & 19.4 & 17,6 \\
\hline Design Speed & $\mathbf{1 2 . 0}$ & $\mathbf{1 4 . 0}$ & $\mathbf{1 8 . 0}$ & $\mathbf{1 6 . 0}$ & $\mathbf{1 6 . 5}$ & $\mathbf{1 7 . 0}$ \\
Max speed & $\mathbf{1 4 . 0}$ & $\mathbf{1 7 . 0}$ & $\mathbf{1 9 . 0}$ & $\mathbf{2 2 . 0}$ & $\mathbf{2 3 . 0}$ & $\mathbf{2 2 . 0}$ \\
\hline
\end{tabular}

Table 4.4: Average speed per vessel class over ten runs. Last two rows indicate the design speed and max speed of the corresponding vessel classes. F is Feeder, $\mathrm{P}$ is Panamax. 
vice speed is higher than design speed. The larger Panamax vessel classes generally have high average speeds. For the WorldSmall and AsiaEurope, we can see in Table 4.3 that we have excess fleet and by comparing $\mathbf{D}(\mathbf{v})$ and $\mathbf{D}(|E|)$ it can be seen that it is mainly the large vessel classes that are undeployed. This is somewhat surprising as this contradicts the economy of scale of larger vessels. However, Table 4.4 also shows that the WorldSmall and AsiaEurope operate at very high speeds for the large vessel classes. An explanation could be the fact that we cannot swap vessel classes very well in the algorithm and we are perhaps not able to fill the larger vessels because we have very good utilization on the small services. This needs further investigation.

\begin{tabular}{|c|c|c|c|c|c|c|c|c|c|c|}
\hline Instance & & $|\mathbf{R}|$ & $\begin{array}{r}\mathbf{t t}(\mathbf{k}) \\
(\%) \\
\end{array}$ & $\begin{array}{r}\mathbf{C}(\mathbf{k}) \\
(\%)\end{array}$ & $\begin{array}{r}\text { tt, } \mathbf{C}(\mathbf{k}) \\
(\%)\end{array}$ & $\begin{array}{r}\mathbf{L}(\mathbf{k}) \\
(\%)\end{array}$ & FFE & $\begin{array}{r}\operatorname{tt}(\mathrm{v}) \\
(\%)\end{array}$ & $\begin{array}{l}\mathbf{C}(\mathbf{v}) \\
(\%)\end{array}$ & $\begin{array}{r}\mathbf{t t}, \mathbf{C}(\mathbf{v}) \mathbf{L}(\mathbf{v}) \\
(\%) \quad(\%)\end{array}$ \\
\hline \multirow[t]{2}{*}{ Baltic } & $\mu$ & 10 & 0.0 & 208 & 0.0 & 79.2 & 653 & 0.0 & 66.4 & $0.0 \quad 33.6$ \\
\hline & & 1 & 0.0 & 6.7 & 0.0 & 6.7 & 57 & 0.0 & 6.8 & $0.0 \quad 6.8$ \\
\hline \multirow[t]{2}{*}{ WAF } & $\mu$ & 7 & 3.4 & 16.2 & 0.0 & 80.4 & 489 & 2.8 & 28.1 & $\begin{array}{ll}0.0 & 69.1\end{array}$ \\
\hline & O & 2 & 7.4 & 12.5 & 0.0 & 9.3 & 230 & 6.9 & 28.4 & $0.0 \quad 26.1$ \\
\hline \multirow[t]{2}{*}{ Med } & $\mu$ & 113 & 32.9 & 0.7 & 5.1 & 61.2 & 1,590 & 41.9 & 0.7 & $7.4 \quad 50.0$ \\
\hline & $\sigma$ & 25 & 11.1 & 0.9 & 3.1 & 12.2 & 521 & 13.0 & 1.2 & 5.114 .6 \\
\hline \multirow[t]{2}{*}{ Pacific } & $\mu$ & 197 & 48.7 & 6.7 & 18.2 & 26.3 & 3,500 & 35.6 & 25.8 & 31.2 \\
\hline & $\sigma$ & 20 & 10.3 & 3.1 & 10.3 & 7.9 & 975 & 11.9 & 12.9 & 17.9 \\
\hline \multirow{2}{*}{ WorldSmall } & & 258 & 37.1 & 31.4 & 17.6 & 13.9 & 12,591 & 41.3 & 26.7 & 22.9 \\
\hline & $\sigma$ & 37 & 12.2 & 8.3 & 7.1 & 14.9 & 2,060 & 13.3 & 6.9 & 9.4 \\
\hline \multirow{2}{*}{ AsiaEurope } & & 823 & 37.7 & 9.8 & 23.4 & 29.2 & 8,918 & 44.4 & 14.9 & $\begin{array}{ll}26.5 & 14.2\end{array}$ \\
\hline & $\sigma$ & 144 & 9.7 & 4.6 & 5.8 & 7.1 & 1,828 & 13.8 & 7.6 & $8.9 \quad 4.8$ \\
\hline
\end{tabular}

Table 4.5: Statistics on the rejected demand reporting average $(\mu)$ and standard deviation $(\sigma)$ over ten runs. $|\mathbf{R}|$ is the number of rejected OD pairs; $\mathbf{t t}(\mathbf{k})$ is the percentage of OD pairs rejected due only to transit time; $\mathbf{C}(\mathbf{k})$ is the percentage of OD pairs rejected due only to lack of capacity; $\mathbf{t t ,} \mathbf{C}(\mathbf{k})$ is the percentage of OD pairs rejected due to both transit time and lack of capacity; $\mathbf{L}(\mathbf{k})$ is the percentage of OD pairs not connected; FFE is the volume of the rejected demand; $\mathbf{t t}(\mathbf{v})$ is the percentage of the volume rejected due only to transit time; $\mathbf{C}(\mathbf{v})$ is the percentage of the volume rejected due only to lack of capacity; tt, $\mathbf{C}(\mathbf{v})$ is the percentage of volume rejected due to both transit time and lack of capacity; $\mathbf{L}(\mathbf{v})$ is the percentage of volume rejected because $\mathrm{O}$ and $\mathrm{D}$ are not connected.

Table 4.5 gives statistics on the rejected demand in the solutions. The primary causes are that existing paths do not meet transit time restrictions, that there is no residual capacity or that the OD pair is not connected in the graph. For the smaller instances (Baltic, WAF, and Mediterranean) rejection of demand is primarily because the OD pairs are not connected, indicating that it is unprof- 


\begin{tabular}{|c|c|c|c|c|c|c|}
\hline \multirow[b]{2}{*}{ Instance } & \multirow[b]{2}{*}{$\begin{array}{r}\mathbf{t t} \\
\text { (days) }\end{array}$} & Objective & \multicolumn{2}{|c|}{ Deployment } & \multirow{2}{*}{$\begin{array}{r}\text { Cargo } \\
\text { flow } \\
\mathbf{T}(\mathbf{v}) \\
(\%)\end{array}$} & \multirow{2}{*}{$\begin{array}{r}\mathrm{CPU} \\
\text { time } \\
(\mathbf{S})\end{array}$} \\
\hline & & $\mathrm{Z}(7)$ & $\begin{array}{r}\mathrm{D}(\mathrm{v}) \\
(\%)\end{array}$ & $\begin{array}{r}\mathbf{D}(|E|) \\
(\%)\end{array}$ & & \\
\hline WorldSmall & +0 days & $-2.80 \cdot 10^{7}$ & 81.9 & 88.4 & 90.2 & 3 \\
\hline WorldSmall & +1 days & $-3.06 \cdot 10^{7}$ & 85.3 & 90.2 & 92.0 & 3 \\
\hline WorldSmall & +2 days & $-3.29 \cdot 10^{7}$ & 88.0 & 91.3 & 92.7 & 3 \\
\hline WorldSmall & +3 days & $-3.43 \cdot 10^{7}$ & 86.4 & 90.2 & 92.9 & 3 \\
\hline WorldSmall & +4 days & $-3.69 \cdot 10^{7}$ & 86.3 & 91.1 & 93.3 & 3 \\
\hline WorldSmall & +5 days & $-3.62 \cdot 10^{7}$ & 89.0 & 93.0 & 94.3 & 3 \\
\hline WorldSme & unlimited (RCSP) & $-3.17 \cdot 10^{7}$ & 92.7 & 94.1 & 94.0 & time \\
\hline WorldSmall & unlimited (Dijkstra) & $-3.51 \cdot 10^{7}$ & 91.7 & 93.3 & 94.3 & time \\
\hline
\end{tabular}

Table 4.6: Influence of extended transit time restrictions for all cargoes. Average of 10 runs. The additional number of days allowed for each cargo (tt). Weekly profit (objective value) $\mathbf{Z}(\mathbf{7})$; percentage of fleet deployed: as a percentage of the total volume $\mathbf{D}(\mathbf{v})$, and as a percentage of the number of ships $\mathbf{D}(|E|) . \mathbf{T}(\mathbf{v})$ is the percentage of total cargo volume transported and $(\mathbf{S})$ is the CPU execution time in hours.

itable to call these ports. For the larger instances (Pacific, WorldSmall, and AsiaEurope) the demand is primarily rejected due to the transit times that cannot be met (with some variation), and in WorldSmall a significant amount of cargo is also rejected due to lack of capacity. In general comparing the percentage not connected in number of demands $(\mathrm{k})$ compared to the volume $(\mathrm{v})$ not connected indicates that it is the demands with low volume that are not connected. Often these demands are from small feeder ports not visited by the solution because the total volume is very low and it is deemed unprofitable by the algorithm.

\subsection{Sensitivity}

Table 4.6 shows the influence of increasing the transit time restrictions given in the Liner-lib with up to five days for all cargos. The results show that the transit time restrictions imposed for each individual cargo are critical. In practice it will not be acceptable to extend all cargo transit times with many days, but as seen even an extension of two days increase the profit of the generated networks by roughly $10 \%$. The results also illustrate how automatic decision 
support tools can help quickly assess the influence of the offered level of service. Likewise, it can serve as a real-time decision support tool to find improvements when evaluating changes in operating conditions or testing different scenarios based on e.g. different service parameters. In addition to the results with an additional five day allowance on the transit time, we have tested the algorithm with unlimited transit time in two settings. When there is no restriction on the transit time, it is possible to solve the shortest path problem using both the resource constrained shortest path, RCSP, algorithm and the Dijkstra algorithm. The performance of the overall algorithm when the Dijkstra algorithm is used is superior, as the sub-problem is solved more efficiently leading to more evaluations and consequently a better network. As seen there is generally significant savings by extending the allowed transit time. Interestingly, the results also show that solving the cargo routing problem with the limits on transit time relaxed by four or five days leads to more profitable networks than when considering unlimited transit time within the allowed computational running time as the shortest path problem is solved faster when transit time is tightly limited. The solution space of the cargo routing problem is limited sufficiently to make the algorithm converge faster than when solving the unrestricted problem, which is in accordance with Karsten et al. (2015).

The left part of Table 4.7 shows the performance of the algorithm for different running times. The results underline that when the algorithm is used to design a new network from scratch, the solution does improve by $10-20 \%$ for the larger instances when running for extended periods, but close inspection of the results also show that the improvements are tailing off. Since the algorithm is intended as an improvement heuristic in practice, the results will be different here. When used as a decision support tool to make incremental changes of a network already in place, the initial solution (the existing network) will be of higher quality than the initial networks considered here, which are constructed by a simple heuristic.

\subsection{Time and Transshipment Constrained Net- work Design}

The transshipment operations are both expensive and time consuming as they require to unload, store, and re-load the container at the intermediate port. Additionally, having fewer transshipments generally reduces handling time, possibly transit time, the risk of damage, and also the risk of missing connections, which is beneficial from a customer perspective. Consequently, transporta- 


\begin{tabular}{|c|c|c|c|c|c|c|c|c|c|}
\hline \multirow{5}{*}{ Instance } & \multicolumn{4}{|c|}{$\begin{array}{l}\text { Transit time } \\
\text { restrictions }\end{array}$} & \multicolumn{5}{|c|}{$\begin{array}{l}\text { Transit time restrictions and } \\
\text { limited transshipments }\end{array}$} \\
\hline & & & Cargo & $\mathrm{CPU}$ & & & & Cargo & $\mathrm{CPU}$ \\
\hline & Obj. & Deployment & flow & time & Obj. & Deplo & yment & flow & time \\
\hline & $\mathbf{Z}(7)$ & $\mathrm{D}(\mathrm{v}) \mathrm{D}(\mathrm{E})$ & $\mathbf{T}(\mathbf{v})$ & $(\mathbf{S})$ & $\mathrm{Z}(\mathbf{7})$ & $\mathrm{D}(\mathrm{v})$ & $\mathrm{D}(\mathrm{E})$ & $\mathbf{T}(\mathbf{v})$ & (S) \\
\hline & $(\mathrm{M} \$)$ & $(\%) \quad(\%)$ & $(\%)$ & (h) & $(\mathrm{M} \$)$ & $(\%)$ & $(\%)$ & $(\%)$ & (h) \\
\hline Pacific & 4.62 & 92.2 & 92.1 & 1 & 5.10 & 92.2 & 88.7 & 91.0 & 1 \\
\hline Pacific & 3.68 & 95.0 & 93.8 & 4 & 3.73 & 95.3 & 82.0 & 93.4 & 4 \\
\hline WorldSmall & -28.0 & 81.9 & 90.2 & 3 & -25.8 & 85.2 & 91.3 & 90.4 & 3 \\
\hline WorldSmall & -31.0 & 84.4 & 91.5 & 12 & -31.1 & 85.5 & 91.2 & 91.6 & 12 \\
\hline AsiaEurope & -14.5 & 84.8 & 88.4 & 4 & -9.80 & 85.6 & 91.9 & 86.7 & 4 \\
\hline AsiaEurope & -17.7 & 86.9 & 89.9 & 16 & -14.4 & 86.2 & 93.0 & 88.7 & 16 \\
\hline
\end{tabular}

Table 4.7: Influence of extended CPU running time and additional level of service requirements. The level of service is considered in terms of restrictions on transit time (left) and restrictions on transit time and the number of transshipments limited to two (right). Average of 10 runs. Weekly profit in million dollars (Obj.) $\mathbf{Z}(\mathbf{7})$; percentage of fleet deployed: as a percentage of the total volume $\mathbf{D}(\mathbf{v})$, and as a percentage of the number of ships $\mathbf{D}(\mathbf{E})$. $\mathbf{T}(\mathbf{v})$ is the percentage of total cargo volume transported and $(\mathbf{S})$ is the CPU execution time in hours.

tions with fewer transshipments are preferred and some customers may actually specify a maximum number of transshipments for their containers, especially for hazardous and high-value cargo. From a carrier perspective, having fewer transshipments also reduces the non-value adding steps and handling capacity requirements, but transshipment operations are important since they permit using vessel capacities more effectively.

The path flow formulation of the multi-commodity flow problem used to solve the cargo routing problem can easily be extended to also explicitly limit the number of transshipments by introducing a resource that keeps track of these. Here, we extend the algorithm for solving the cargo routing problem to include restrictions on the number of transshipments by including an additional resource. The additional resource is incremented by one at transshipment edges and included in the dominance criterion in addition to transit time. Furthermore, paths that violate either the transshipment or transit time criterion are deleted.

Table 4.7 reports results for the network design algorithm, and as seen the solutions are still profitable when including a limit on the number of transshipments in addition to the transit time requirement. We impose that all cargoes cannot transship more than twice as this is the case in practice for 
more than $95 \%$ of the cargo. However, for some cargoes this will constrain the solution space too much, but we do not have more detailed information on the number of allowed transshipments available. The results for the shorter running times show that some cargo that was previously transported is now rejected and the objective values are approximately $10 \%$ worse for Pacific and WorldSmall, whereas they are approximately $30 \%$ worse for AsiaEurope. Further analysis reveals that solving the cargo routing problem with tight limits on transit time, and the number of transshipments limited to two, in terms of computational time is comparable to or slightly faster than when only considering transit time. So even though the shortest path algorithm is extended with an additional resource, the solution space of the cargo routing problem is limited sufficiently to make the algorithm converge faster. Hence, the level of detail considerations can help restrict the solution space sufficiently to offset the increased complexity of the label setting algorithm and turns out to improve dominance significantly. However, on the input side the number of transshipments allowed for each cargo will influence the performance. So even though the solution time of the sub-problem does not increase, it is harder for the network design algorithm to find good solutions. When we increase the allowed running time of the algorithm the picture changes as seen in Table 4.7 The profitability of the created networks with a better level of service is the same as the networks where only transit time is restricted. For the WorldSmall instance the profit of the network created only with transit time restrictions is $31 \mathrm{M} \$$, while the profit is $31.1 \mathrm{M} \$$ when considering both transit time restrictions and limited transshipments. This is a very encouraging result. We only show results for larger instances where some of the cargo is transshipping more than once (for the smaller instances all cargo is transported using direct on one-transshipment connections). Furthermore, Table 4.7 illustrates that transit time and number of transshipments are correlated, and generally limiting one of them will implicitly bound the other, e.g. a container that has a transit time requirement of one week can only transship very few times before the allowed transit time is violated. Likewise, it is only possible to reach a limited part of the network if a customer only allows one transshipment. In this case the transit time for a container will be bounded by the longest of the possible direct and one-transshipment connections. The ability to control the number of transshipments gives a way of offering products to the customers that are more tailored to their needs. Being able to offer fewer transshipments may attract new customers even though these products are offered at a higher price and it will be possible during the network design process to consider differentiated products between any pair of ports in terms of offered transit time and number of transshipments. Likewise, it is easy to get an overview of possible routings with more transshipments that can be offered at a lower price while helping to ensure a better utilization of the network. 


\subsection{Conclusions}

We have presented a model for the TCLSNDP introducing transit time restrictions on each individual commodity while maintaining the ability to transship between services. We have extended the matheuristic of Brouer et al. (2014b) to handle the new constraints. The core component of the matheuristic is an integer program considering a set of removals and insertions to a service. We extend the integer program to consider how removals and insertions influence the transit time of the existing cargo flow on the service. Each iteration of the matheuristic provides a set of moves for the current set of services and fleet deployment, which lead to a potential improvement in the overall revenue. The evaluation of the cargo flow for a set of moves requires solving a time constrained multi-commodity flow problem, which we solve using column generation.

The introduction of transit time constraints changes the estimation functions for the improvement heuristic and the pricing problem of the column generation algorithm from an ordinary shortest path problem to a resource constrained shortest path problem. We apply the specialized label setting algorithm of Karsten et al. (2015) to achieve satisfactory performance. Additionally, we show that it is tractable to extend the algorithm to consider a limit on the number of allowed transshipments as we can still create profitable networks of comparable quality.

Extensive computational tests show that it is possible to generate highly profitable networks for the majority of the instances in Liner-lib when considering level of service requirements and, especially for the larger instances, the approach generates networks of good quality. Furthermore, the tests show that from a computational perspective it can even be advantageous to include level of service requirements. However, some demand is not served and the fleet is not utilized completely, especially for the larger vessel classes, suggesting that further algorithmic improvements may lead to even better solutions. In particular, we expect that speed optimization on individual legs as well as more flexibility in terms of possible vessel class swaps could improve the algorithmic performance.

\section{Acknowledgments}

This project was supported by the Danish Maritime Fund under the "Competitive Liner Shipping Network Design" project. 


\section{Bibliography}

Agarwal, R. and Ergun, O. (2008). Ship scheduling and network design for cargo routing in liner shipping. Transportation Science, 42(2):175-196.

Álvarez, J. F. (2012). Mathematical expressions for level of service optimization in liner shipping. Journal of the Operational Research Society, 63(6):709-714.

Archetti, C. and Speranza, M. G. (2014). A survey on matheuristics for routing problems. EURO Journal on Computational Optimization, 2:223-246.

Brouer, B., Álvarez, J., Plum, C., Pisinger, D., and Sigurd, M. (2014a). A base integer programming model and benchmark suite for liner shipping network design. Transportation Science, 48(2):281-312.

Brouer, B., Desaulniers, G., and Pisinger, D. (2014b). A matheuristic for the liner shipping network design problem. Transportation Research Part E: Logistics and Transportation Review, 72:42-59.

Christiansen, M., Fagerholt, K., Nygreen, B., and Ronen, D. (2013). Ship routing and scheduling in the new millennium. European Journal of Operational Research, 228(3):467-483.

Gelareh, S., Nickel, S., and Pisinger, D. (2010). Liner shipping hub network design in a competitive environment. Transportation Research Part E: Logistics and Transportation Review, 46(6):991-1004.

Karsten, C. V., Pisinger, D., Ropke, S., and Brouer, B. D. (2015). The time constrained multi-commodity network flow problem and its application to liner shipping network design. Transportation Research Part E: Logistics and Transportation Review, 76:122-138.

Liu, Z., Meng, Q., Wang, S., and Sun, Z. (2014). Global intermodal liner shipping network design. Transportation Research Part E: Logistics and Transportation Review, 61:28-39.

Meng, Q., Wang, S., Andersson, H., and Thun, K. (2014). Containership routing and scheduling in liner shipping: Overview and future research directions. Transportation Science, 48(2):265-280.

Mulder, J. and Dekker, R. (2014). Methods for strategic liner shipping network design. European Journal of Operational Research, 235(2):367-377.

Notteboom, T. E. and Vernimmen, B. (2009). The effect of high fuel costs on liner service configuration in container shipping. Journal of Transport Geography, 17(5):325-337. 
Plum, C., Pisinger, D., and Sigurd, M. M. (2014). A service flow model for the liner shipping network design problem. European Journal of Operational Research, 235(2):378-386.

Reinhardt, L. B. and Pisinger, D. (2012). A branch and cut algorithm for the container shipping network design problem. Flexible Services and Manufacturing Journal, 24(3):349-374.

Stopford, M. (2009). Maritime Economics. Routledge, third edition.

Wang, S. and Meng, Q. (2014). Liner shipping network design with deadlines. Computers and Operations Research, 41(1):140-149.

Wang, S., Meng, Q., and Sun, Z. (2013). Container routing in liner shipping. Transportation Research Part E: Logistics and Transportation Review, 49(1):1-7. 